\title{
Swear Words among Males: The Social Functions and Pragmatic Meanings
}

\author{
Slamet Setiawan \\ Universitas Negeri Surabaya \\ Surabaya, Indonesia \\ slametsetiawan@unesa.ac.id
}

\author{
Fatimatuzzahroh \\ Universitas Negeri Surabaya \\ Surabaya, Indonesia \\ fatimatuzzahroh@mhs.unesa.ac.id
}

\begin{abstract}
Swearing at some stage is similar to cursing; a linguistic activity utilizing taboo words to express strong emotions. Traditionally, swear words contain taboo and offensive expressions. Thus, the users are negatively judged and labeled. Are swear words always negatively framed? This paper tries to prove that such expressions do not only convey negative sense but also provide diverse functions and meanings. Dual mode of communicative data was gained; spoken and written. Besides, direct and virtual interviews were conducted to reveal the males' underlying reasons for swearing. The results may indicate that the use of swear words carries social functions, namely: intraindividual and interindividual which are reflected into cathartic, abusive, emphatic, idiomatic, and dysphemistic swearing. Nonetheless, cathartic and abusive searing are mostly committed. Another finding, pragmatically, swears words are bound by context. They can reflect not only the user's anger but also happiness and even solidarity. The other finding is that males swear more frequently in the presence of a group consisting of their own gender. All these evidence might be a gentle warning on the effect of what one produces and the wise interpretation upon one produces. When these are taken into account, living in harmony is created.
\end{abstract}

Keywords—swearing, swear word, gender, social function, pragmatic meaning

\section{INTRODUCTION}

Swearing is a natural human behavior which is practiced in almost all languages in the world. Each language, however, has its own words or expressions to swear. In English, the term of the swear word denotes a particular type of linguistic [1]. This is a linguistic phenomenon that is worth studying and examining. In most society, it is forbidden to use swear word freely in public but the expressions still exist. Swear word contains two level: institutional and individual. These levels are restricted and sanctioned as harmless if the swear words are spoken [2]. This kind of thought gives some restrictions to the use of swear words in public. The users of swear words are judged and disrespected by the societies because their speech contains offensive words.

An institutional level, the restriction is broader and harder to tolerate because it deals with the individual in public, while each individual has his or her own preferences about swear words. However, in the individual level, it is easier to tolerate the use of swear words, since it only faces a single person and fewer preferences. Moreover, the majority of societies in
Indonesia do not allow the use that expletives word in public, because according to norms, speaking by using swear word is counted to be a bad or impolite person. However, there is no linguistic form that inherently imbued with politeness and impoliteness, due to it manifestoes with politeness within a given community of practice [3]. So, the impoliteness that the societies mark to the swear word user is because they are not from the same community or culture.

Swear word is literally a word containing expletive, taboo, dirty, vulgar or offensive word. In the American heritage dictionary of the English language (2000), this term refers to a lexicon of offensive emotional language [2]. Another definition; it is undereducated, obscene, rude, and profane language in society [4]. The interesting phenomenon is that swear word in most of the cases always carries offensive meaning, however, the societies still use it regularly. Although it always carries expletives meaning, there must be interesting and unique meaning that swear word can present [4].

Swearing, almost a hundred percent, presents the speakers' emotion which carries various meanings depending on the situation and context of the event. There are five types of swearing, namely: dysphemistic, abusive, idiomatic, emphatic, and cathartic swearing [5]. Dysphemic swearing has an effect that makes the listener think about negative thing or matter (That's just bullshit!). Abusive swearing is used to abuse or insult someone (Fuck you bitch!). The next is idiomatic swearing that is used to show off or arouses an interest (Fuck, man). The fourth is emphatic swearing that is used to emphasize something (Wow, it is so fucking great). The last type is cathartic swearing which is used to show the bad impact of bad thing has happened (Damn this coffee!).

The use of emphatic swearing is mostly used among teenagers and boys since the function is to emphasize something that they want to express but there is no linguistic form that can represent their feeling. The focus of this paper is boys since they use swear words more than girls. It is claimed that men use the more offensive word (i.e. fuck, shit, motherfucker) than women do [2]. Men produce $67 \%$ swearing episodes in 1986 and narrowed to $55 \%$ in 2006 while women only produce less than a half of it, that is $24 \%$ expletives word in 2006 data. 
The similar topic as this present study examined male's and female's attitudes towards swear words [5]. This paper revealed the international secondary students' attitudes toward the swear words of their form, type, meaning, and function. Since the informants of the previous study were bilingual students, the swear words that they produced were in two languages; Indonesia and English. The results show that female and male students used swear words in two languages. Even though the setting of the study was in Indonesia, in fact, they used more English language swear words than Indonesian. The male students used four Indonesian and six English swear words, while the female respondents used only two Indonesian swear words and eight English swear words. The word "fuck" was the most used to swear by both genders. It was also revealed that the function and meaning of swearing in this study were only to shows literal meaning. They used swear words in their speeches in order to release their stress and express intense emotions.

Unlike the previous study, this present study tries to disclose swear words further seen from the social function and pragmatic meaning. When someone does swearing, it is not easy to conclude its function and meaning. It might carry two possibilities: explicit or implicit meaning. Thus, this paper intends to seek some aspects, namely: types of swear words; under what circumstances the swear words used; informants' reasons for using swear words; and informants' attitudes when they are swearing in presence of females.

The following is a brief discussion on theories which link to the stated title and research questions.

\section{A. Pragmatic Paractice and Meaning}

Pragmatics has a correlation between language, meaning, and use. The meaning of a certain expression can be beyond the sentence or speech of a given language. People in society tend to apply pragmatic practice almost in their daily conversation regardless of the gender. Pragmatic practice can occur in a very simple conversation as in (01) and (02).

\section{(01) A: I am cold.}

The utterance "I am cold" from somebody in a room with the on air-conditioner may have more than a single reading. The meaning of this expression is not only somebody's expression of the cold temperature $\mathrm{s} / \mathrm{he}$ felt but also a request $\mathrm{s} / \mathrm{he}$ made to someone else to turn off the air conditioner. Similar to this, study the expression in (02).

(02) A: I am hungry.

The "I am hungry" expression also has the same case. It is not about the hunger feeling of the speaker, but it may be interpreted as an invitation to the speaker's interlocutor to go to the canteen or restaurant to get some food to heal the hunger feeling. It is clear that the meaning of a particular expression is based on the context and situation. In fact, pragmatic practice does not always occur in natural-daily conversation, but also in swearing.
Many linguists argue that swearing falls into the pragmatic territory rather than semantic [6]. The matter thing of utterances is not only the natural syntax of utterances, but also the manner in which the utterances are delivered. It should reflect what the speaker's intention to say. The meaning can be explained through the communicative situation when the terms are uttered. This situation refers to pragmatic utterance or communicative functions [1]. The claim that swearing also consists of pragmatic meaning is similar to the idea that people learn to judge when, where and whom it is appropriate to swear or where swearing would be offensive [7]. Thus, it is obvious that the relation between speaker and listener as well context plays important role in creating the pragmatic meaning in swearing.

Another fact that swear word contains pragmatic meaning is interjection. This reflects a reaction of the speaker from a sudden or unexpected accidents whereby it can be detailed as reactive expletive interjections. This interjection does not have one meaning to establish the term. It has probability more than one meaning, it could be anger, joy, protest, surprise etc. [4]. Study the following excerpt in (03).

(03) A : I'll have the yellow ones.

$\mathrm{B}$ : The yellow ones?

A: Just <pause> Oh bloody hell!

$B$ : The yellow ones were thrown.

A: What do I do?

The utterance "Oh bloody hell!" indicates the disappointment feeling of the speaker due to the stuff that speaker A looked for is no longer in the cupboard [4].

Another example is when someone accidentally stubbed his or her finger with a nail and yelled "Fuck" directly, this word shows the expression of pain from the speaker not to isolate someone else. The word "fuck" actually has a lot of meaning. It can be to isolate or to spread hate feeling or anger as well, however, in this case, it shows the pain expression of the speaker himself. This is reactive expletive interjections since it comes out from the speaker as the first reaction of the sudden accident. On the other hand, the use of word "fuck" can be different if the speaker in crowded and there is someone accidentally stub her or his hand with a nail. The word "fuck" will have offensive and rude meaning as isolation or anger. Moreover, the pragmatic meaning also occurs in emphatic function. The speaker uses swear word to emphasize his feeling about something. Emphatic function helps the speaker to get their message across [4].

\section{B. Function of Swear Word}

In swearing people have their own intention. Normally it has a negative emotion of the speaker [4]. It can be used to isolate someone because the hate feeling or to attack someone. In this case the speaker tends to choose swear word that contains offensive or rude word. Offensive and rude word are the best choice to isolate or mock someone, due to it can represent the speaker's feeling using that swear word. In this case, the function of a swear word is as an abusive function. It could also represent an excited feeling or emphatic function [5]. Swearing 
can be differentiated by its particular function [8]. At least there are two: annoyance and social swearing. The former serves the primarily intra-individual functions (e.g., catharsis) while the latter serves inter-individual swearing [9] [8].

The effect of cathartic has relation with the intra-individual function since the swear words are used to let one's emotion; it can be anger or pain. Cathartic swear word has the ability to reduce pain. One of the studies demonstrated to ask the informants to put their hands in the ice of water while they had to choose and repeat between the chosen swear or neutral word [10]. The result shows that the participants who repeated swear word could endure the painful stimulus longer than uttering the neutral word. On the other hand, inter-personal function deals with the people's thought about the swear word and the speaker. Since the swear word is judged by people as offensive and taboo language, the speakers are often considered to be antisocial and offensive. Consequently, swearing can give impact to the speaker's individual status and how the speaker is perceived by others [8]. A further inter-individual function of swearing depends upon the specific interpersonal context, including the composition of the group in which swearing occurs.

At least there are five ways of swearing: descriptively (Let's fuck), idiomatically (It's fucked up.), abusively (Fuck you, motherfucker!!), emphatically (This is fucking amazing!), and cathartically (Fuck!!!) [5].

\section{METHOD}

This study employed a qualitative method using interview technique. Ten male subjects were involved: five of them were university students and the other five were male netizens who were taken from Instagram. All informants were randomly selected. Note to be made here that all informants are males. This is the major concern in this study as males, according to many studies, are found to be ones who often do swearing [7]

(04)

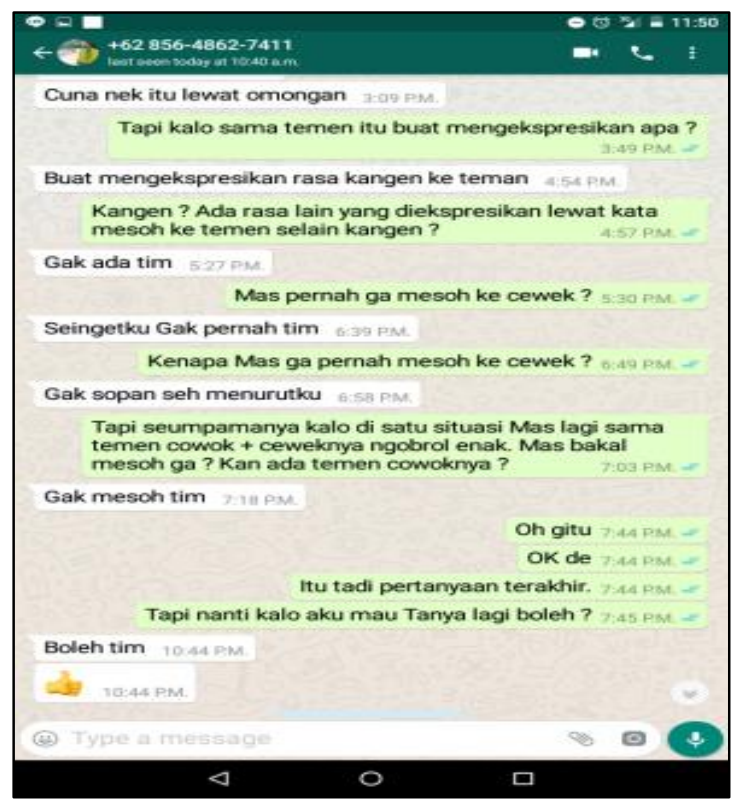

Fig.1. Some reasons of swearing
The interview was conducted by using two modes: direct and indirect interview. The former was conducted by face to face interview involving three informants of the university students, whereas the latter was done by WhatsApp involving seven informants as seen in Figure 1. Every informant was given four questions related to the swear words.

It is good to share that recruiting informants was quite challenging. Most of them were reluctant to participate as soon as they knew that the topic was about a swear word. One university student rejected to be interviewed. The request was sent to more than ten netizens but only five replied to the given interview questions. The evidence of the interview via WhatsApp is like in (04) or in Figure 1.

\section{RESULTS AND DISCUSSION}

\section{A. Type of Swear Word Used Among Males}

Focusing on male participants in this study is because males use more swear word rather than girls. One study confirmed this claim when they are angry and frustrated [7]. Another study also supports the claim that men and women swear at the same rate but men use more offensive word than women [7]. Men also tend to use that swear word almost in all sentences and situations of their life.

Swear word that males usually use can be described from various categories and be associated as the following: sex organs; sexual activity; animal names; mental illness including stupidity [11].

TABLE I. SWEAR WORD CATEGORIES

\begin{tabular}{|c|c|c|c|}
\hline Sex organs & Sexual activities & Animal names & Others \\
\hline $\begin{array}{l}k-n t h-l=4 \\
\text { 'ball' } \\
j-m b-t=1 \\
\text { 'pubic hair' }\end{array}$ & $\begin{array}{l}j-n c-k(c u k)=9 \\
\text { 'fuck' } \\
\text { fuck }=4\end{array}$ & $\begin{array}{l}\text { anjing = } 6 \\
\text { 'dog' } \\
\text { jangkrik = } 3 \\
\text { 'cricket' lit. }\end{array}$ & $\begin{array}{l}\text { taek }=5 \\
\text { 'shit' } \\
\text { bullshit }=1 \\
\text { shit }=1 \\
\text { bitch }=1\end{array}$ \\
\hline
\end{tabular}

Table I above presents the swear words that are listed from the most to less offensive and the evidence of an informant's response. The word $j-n c-k$, anjing, taek, fuck, and $k-n t h-l$ are chosen as the top five words that informants usually used. It shows that the word $j-n c-k$ (cuk) or 'fuck' in English under the sexual activity category is the most used swear word which is reported by nine informants. Followed by anjing is chosen by six participants; taek were taken by five participants while "fuck" and $k$ - $n$ th- $l$ has been favored by the same number of participants, four.

Some of the participants said that they used the word $j-n c-k$ (cuk) almost in all conversations. One of my participants reported that he preferred to use short form $c u k$ because he has been comfortable with it. For the word anjing, some participants said that they used this word because it can be used to curse someone. This word can be used to regard the interlocutor as the animal or anjing ('dog' in English) which has a negative sense. The most cases the function of the word 
anjing is to release anger or emotion of the speaker. Moreover, the word taek 'shit' was reported to be used to make a joke to someone. The degree of rudeness or offensiveness of this word is not as high as the word $j-n c-k$ and anjing. Besides, this expression can be used to isolate or curse someone else, however, the impact of the curse is not as strong as anjing.

The next swear words are "fuck" and $k$-nth-l. These words can be used to isolate or curse someone, but it is weak form. However, these words can also be used when there is bad thing happen or cathartics. While the remaining four swear words $j$ $m b$ - $t$, "bitch", "shit", and "bullshit" have the weakest level of rudeness and offensiveness.

When anger is taken into account, men and women swear at the same rate but men use more offensive word than women [7]. Moreover, one study revealed that sex-related insults are common across cultures [12]. This study also claims that men use more offensive swear word to convey or use in their swearing, and the use of sexual activity is the most used to swear.

\section{B. Situation of Swearing}

Swearing for the majority of people consist of taboo language, that may contain the speaker's emotion. Swearing is a taboo language that the purpose is to express the speaker's emotional state and deliver the information to the listener [7]. Study the data in Table II.

TABLE II. SiTUATION OF SwEARING

\begin{tabular}{|l|l|}
\hline \multicolumn{1}{|c|}{ Situations } & \multicolumn{1}{c|}{ Frequency } \\
\hline Get angry & 8 \\
\hline Get mad & 8 \\
\hline Chill out with friend & 5 \\
\hline Joking (laugh for something funny) & 4 \\
\hline Habit & 2 \\
\hline Annoyance & 1 \\
\hline
\end{tabular}

Table II above describes the conditions that lead the participants to do swearing. It can be seen that the top two situations which could lead the participants to do swearing are emotional situation: angry and get mad which were reported by eight participants. The second top situation is to chill out with friend confessed by five participants. The next is a kind of joking (laugh for something funny) that is declared by four participants. Swearing as a habit was reported by two participants. The last situation is to express annoyance reported by only one participant.

Eight of participants mentioned that they did swearing most when they got angry or got mad. They also mentioned that when they swore in angry condition, it could help them to release their anger so that it can make them feel better [7]. This evidence supports the claim that swearing can be used to express a variety of strong feelings, in particular, annoyance, frustration, and anger [1] as cited from Carter and McCarthy. This case is categorized as cathartic swear word which is triggered by a bad thing and it makes their feeling back to neutral. In addition, swearing conveys the strong emotions and regain better feeling. This behavior can be perceived as cathartic rather than rude. This case reflects the function of the the swear word as intrapersonal, since after swearing the speaker can feel relief.

The emotional situation does not always bring the function of cathartics, but it also can be an abusive function provided the swear words are used to attack someone else. This kind of swearing contains the real meaning since swear word is a taboo and offensive language [7]. Some of the participants stated that they also swore when they got angry to attack or mock someone. This case also can let off the emotional feeling of the speaker [5]. In this case, the function of the swear word is interindividual since it deals with the environment and somebody else. The participants swore to attack people, and further the listener would give response according to the swear word that had been used.

Since the general concept of the swear word is offensive and taboo language, the function of this word is not only always about negative emotions but also positive emotion. From Table 2 , it can be seen that the second top situation to swear is to chill out with friends and followed by joking (laugh for something funny). Those two kinds of situation can be concluded into positive emotions such as happiness, excitement, enthusiasm, and surprise [4]. Five participants stated that they used swear word when they chilled out with friends and sometimes used it to have a joke with friends. This, according to them, can make their intimate relation among them grew stronger. Therefore, it is not surprising that the swear words are frequently present in males' conversation. Some of them gave an opinion that employing swear words is considered to be a close friend. This evidence brings the function of a swear word as interindividual and it occurs based on the effect of emphatic swear word.

Sometimes, the speakers emphasize their utterance by using swear word with intention of force about something they want to say. There are some cases which show that habit situation can lead to swearing is because the speakers (two participants) felt comfortable with the swear word, and they also wanted to show to the surrounding that they were cool with the swear words they uttered. Two participants stated that they swore because it could be the self-identity and they felt cool when they swore. This kind of swear word has the function interindividual as reflecting of idiomatic swearing effect. This exists because it is used to arouse interest or to show off [5]. Thus, this kind of swear word is mostly used when the interlocutors are present during communication is in action.

The last situation in which the speaker produces swear word is annoyance situation. Most of this situation is used as selfdefending, however, the function of this situation mostly is inter-individual under the effect of abusive swearing. Abusive swearing is used to attack someone. In this case, the speaker uses swear word in order to make himself free from the annoyance. Participants said he used swearing in this case because he wanted to be alone with his game or even his world. 


\section{Reasons for Swearing}

Swearing has the concept of taboo language that consists of an offensive word. Normally, swear word contains negative meaning that is directly stated in the language. This Table III below shows some reasons why the participants use swear word in such situation that has listed in Table II.

TABLE III. REASON FOR SWEARING

\begin{tabular}{|l|l|}
\hline \multicolumn{1}{|c|}{ Reasons } & \multicolumn{1}{c|}{ Frequency } \\
\hline Release anger and pain & 10 \\
\hline Relation with friends & 7 \\
\hline Emphasis something & 4 \\
\hline Habitual & 2 \\
\hline Self identity & 2 \\
\hline
\end{tabular}

Table III shows some reasons for the participants in swearing. Releasing anger becomes the top reason why swear word is chosen by ten participants. The second place is keeping relation with friends which were reported by seven participants and then is followed by emphasizing thing (by four participants). The last reason is exposing habit and self-identity (by two participants).

This section explains the pragmatic meaning of swearing; reason that contains the pragmatic value. The first reason is releasing anger and pain. From the previous section, it has been addressed that swearing is a taboo expression to express the speaker's emotional state and deliver the information to the listener based on [7]. In releasing the anger, swearing has the direct meaning in which the probability of pragmatic is little. However, there are cases when a person is releasing his anger by utilizing interjection as cathartic function. The expression is no longer offensive or rude word for someone else but it conveys the pain and anger of the speaker himself. This means that it is a spontaneous reaction of the speaker from a sudden or unexpected accident [1]. Someone's expression "fuck", for example, because his thumb hit a wall, contains pragmatic meaning as this conveys more than one reading.

The intended meaning of swearing is mostly based on what the speaker says. For instances, if there is a person whose hand gets stubbed, s/he will spontaneously say "fuck" or in Javanese $j-n c-k$. Those two words have the same literal meaning of "sexual activity" and have the same meaning for swearing; showing rudeness and offensiveness. In addition, to know whether or not swearing has the pragmatic function is when it has a relationship with the manner in which the utterances are delivered. The pragmatic meaning of swearing also has a relationship with whom where and where the swear word utter [7]. This case briefly explains the relationship between speaker and listener which brings the pragmatic meaning in swearing. The following in (05) is the example.

(05) A: Hei cuk! Piye, wis mari tugase?

'Hi fucker! Have you done your assignment?'

B: Wis. Kon cuk, wis mari tah?

'It's done. What about you, fucker? Have you done it?'
The speaker A and B (both are males) used the same swear word $c u k$ which is the short form of $j-n c-k$ (same as fuck). In this case, those two males were swearing at each other, however, the meaning of the swearing is not to attack each other. On the other hand, this swear word conveys meaning beyond the word; this can show the intimacy between them. They want to show that they are very close friends. Seven out of ten participants in this study confessed that they swore to their friends to build intimacy among them. They also admitted that it was more comfortable to use swear word to address their friends. This kind of swearing is counted as non-offensive. The use of swearing between close friends is usually an indication of intimacy or group membership, this way of speaking shows the solidarity and identity [4] [7].

Another swearing which carries pragmatic meaning is presenting self-identity. Two out of ten participants stated that swearing for them are used to show their unique identity. Study the comment from the informant below in (06).

(06) When I do not do swearing, it is just not like me anymore, so that I keep using swear word in my daily conversation.

This is the evidence of swearing which has pragmatic meaning. The meaning of this swear word is broad since the value of selfidentity is only recognized by the speaker himself.

The pragmatic meaning also occurs when the speaker uses swear word to emphasize something [4]. The meaning of the term is no longer offensive or rude. It has changed to be something beyond the word which may describe the feeling of joy or happiness, as presented in (07).

\section{(07) This flower is so fucking beautiful.}

The word "fucking" points to something beautiful and has no sense of rudeness at all. One participant in this study reported that he always uses swear word to emphasize something as the expression of amazement or admiration. From all evidence above, it might not be too exaggerated to say that in swearing, males usually employ pragmatic meaning.

\section{Swearing in the Present of the Other gender}

A taboo expression has different values if it is examined from different perspectives. In a culture where its society holds a value of politeness, this kind of language is forbidden to use. However, in a different culture, the same expression seems to be flexible. Even in girls' perspective, swear word is not one hundred percent forbidden, but it cannot be used often since the concept of this language is taboo and offensive. Table 4 below shows how males' behavior (the informants of this study) related to swearing to their opposite gender.

TABLE IV. SWEARING TO THE OTHER GENDER

\begin{tabular}{|l|c|}
\hline \multicolumn{1}{|c|}{ Swearing to the Other Gender } & Frequency \\
\hline Never swear to girls & 7 \\
\hline Swear to girls (certain reason) & 3 \\
\hline
\end{tabular}


The table IV shows that there are three participants out of ten reported that they used swear word in the presence of girls. The remaining participants (seven) confessed that they never swore to female interlocutors. This may the indication that males are still likely to swear in the presence of the same gender. This finding is similar to the claim that people of both genders are less likely to swear in the presence of a person with a higher status or in the presence of someone of the other gender [8].

In relation to swearing to the opposite gender or in the present of the other gender, one participant stated as in (08).

(08) I will never ever swear to a girl or in front of a girl because it is not appropriate.

The participant seems to realize the factor which influences him not to swear, namely: his interlocutor of the opposite sex. This is in line with the idea that the important factors related to the toleration of swearing namely the presence of the other gender [13].

Generally, swearing is more tolerated in an informal and private situation. This implies that most case as presented in Table 3 that males are not frequently swearing in front of the presence of another gender because another gender may not tolerate their swearing. Males are aware of the consequence that when they are swearing in front of the other gender, they will lose their respect and social status. Therefore, it is plausible that the informant stated as in (08). This is supported the statement that "men and women swear more frequently in the presence of a group consisting only of their own gender than in mixedgender contexts" [8].

\section{CONCLUSION}

There are some categories of swear words used by males: sex organs, sexual activities, the name of animals, and other forms. The most frequent use is sexual activities. Generally, sex-related insults are common across cultures.

Males and females swear at the same rate, however, males use swear word more frequent than females, and the swear words used by males are more offensive. Males are more likely to swear when they get angry and frustrated. It is also found that the participants use more offensive swear word in their daily conversation rather than less offensive ones. It is simply because they have been comfortable in using that swear word and want to curse someone when they are in conversation.

In addition, in swearing, boys also bring the pragmatic meaning, which is shown from the relation between the speaker and listener. The swear words in this particular context do not only convey offensive meaning but also it contains a solidarity. Males tend to use swear word to build intimacy among their friends. Moreover, in swearing boys also carry function such as intraindividual and interindividual as the reflecting of the effect of cathartics, abusive, emphatic, idiomatic, and dysphemistic swearing. The most function that is used by the participants is inter-individual. Meanwhile, intraindividual is reflected by cathartic swearing. In addition, males in swearing mostly represent the effect of cathartic and abusive swearing, since swearing can be used to express the strong emotion of the speaker.

When swearing males tend to consider the presence of females. They frequently do the swearing in the non-presence of the other gender. It is just not appropriate. In fact, females feel uncomfortable when they have to listen to swear word in every single turn in the conversation.

\section{REFERENCES}

[1] M. Ljung, "Swearing: A cross-cultural linguistic study," vol. 8, pp. 1203. 2011.

[2] T. Jay, "Massachusetts College of Liberal Arts," American Heritage, (March), 1-33. 2009. pp. 267-289.

[3] M. Dynel, "Swearing methodologically: the ( im ) politeness of expletives in anonymous commentaries on youtube differences these terms exhibit, according to their dictionary definitions ) tend to be deemed solely as impolite and are even reported to have constitut," Journal of English Studies, vol. 10, pp. 25-50, 2012.

[4] N. Wang, "An analysis of the pragmatic functionsof "swearing" in interpersonal talk," vol. 6, 2013.

[5] M. F. S. Nicolau and K. E. Sukamto, "Male and female attitudes towards swear words: A case study at Binus International School,” K@Ta, vol. 16, no. 2, pp. 71-76, 2014.

[6] C. Goddard, "Swear words and curse words in Australian (and American) English at the crossroads of pragmatics, semantics and sociolinguistics," Intercultural Pragmatics, vol. 12, no. 2, pp. 189-218, 2015.

[7] T. Jay and K. Janschewitz, "The pragmatics of swearing," vol. 4, 2008.

[8] J. J. M. Vingerhoets and L. M. Bylsma, "Swearing: A biopsychosocial perspective," vol. 22, pp. 287-304, 2013.

[9] Montagu, "The anatomy of swearing," Pennsylvania: University of Pennsylvania Press 19104-4011, 1967.

[10] R. Stephens, J. Atkins, and A. Kingston, Swearing as a response to pain. Neuororeport 20, pp. 1056-1060, 2009.

[11] D. M. Mukuni, A. M. Ireri, P. M. N. Thiga, and F. A. Owili, "The social function of swearing in a miraa market environment," Arts and Social Sciences Journal, vol. 7, no. 2, 2016.

[12] M. Drews, "The science of swearing: A look into the human mind and other less socially acceptable four-letter words," vol. 7, 2014.

[13] Daly, Nicola, J. Holmes, J. Newton, and M. Stubbe, "Expletives as solidarity signals in FTAs on the factory," Journal of pragmaticz, vol. 36, no. 5, pp. 945-964, 2004. 